\title{
ESTUDO DA TEMPERATURA SUPERFICIAL DE LAGOAS COSTEIRAS ATRAVÉS DE PARÂMETROS METEOROLÓGICOS E DADOS DE SENSORIAMENTO REMOTO
}

\author{
Silvia Beatriz Alves Rolim ${ }^{1}$, Marilei Bender Xavier ${ }^{1}$, Gervásio Annes Degrazia ${ }^{2}$, \\ Lilian Piecha Moor ${ }^{2}$, Waterloo Pereira Filho ${ }^{3}$ \\ ${ }^{1}$ Programa de Pós-Graduação em Sensoriamento Remoto,Universidade Federal do Rio Grande do \\ Sul, Porto Alegre, RS, Brasil \\ ${ }^{2}$ Universidade Federal de Santa Maria, Departamento de Física, Santa Maria-RS, Brasil \\ ${ }^{3}$ Universidade Federal de Santa Maria, Departamento de Geografia, Santa Maria-RS, Brasil
}

\section{RESUMO}

Este trabalho apresenta um método para estimar a temperatura da superfície de lagoas usando imagens de satélites. A investigação mostra que existe uma relação entre as medidas da temperatura e os padrões de vento.

\section{SUMMARY}

This paper presents a method to estimate the surface temperatures of lagoons using satellite images. The investigation reveals that there is a direct relationship between measured temperature of the lagoon and the wind patterns.

\section{INTRODUÇÃO}

As observações de parâmetros meteorológicos em ambientes naturais podem revelar e predizer o efeito de impactos ambientais em ecossistemas caracterizados por escalas de tempo geológicas curtas. Os ecossistemas aquáticos que possuem pequenas extensões (lagoas e pequenos lagos), são sensíveis às mudanças nos padrões de temperatura (Mohseni et al., 2001), bem como na velocidade do vento e em outras variáveis. O objetivo deste estudo é empregar imagens de satélites para medir remotamente a temperatura superficial (TS) da lagoa Mangueira, que está situada no Sul do Brasil na interface entre o oceano e a região continental de terra firme. Um objetivo adicional do presente estudo é analisar a magnitude e direção do vento e relacionar estas quantidades com as flutuações da TS da 
lagoa Mangueira.

\section{DESCRIÇÃO DA ÁREA DE ESTUDO}

A lagoa Mangueira é um ecossitema aquático constituído principalmente por um corpo de água raso com profundidade variando de 1,5 e 6 metros (Delaney, 1965). Ela apresenta uma forma alongada com $92 \mathrm{~km}$ de comprimento e uma largura variando entre $2 \mathrm{e}$ 10 km e esta localizada entre as cidades de Santa Vitória do Palmar e Rio Grande.

\section{MÉTODOS E MEDIDAS DE VENTO E DA TS}

Uma variedade de artigos científicos discute e analisa a variação da temperatura superficial de diferentes alvos. Vários sensores, abordo de satélites, possuem dados na região do infravermelho termal. Um desses sensores é o Espectro Radiômetro Imageador de Resolução Moderada (MODIS) do satélite EOS-TERRA. Entre os vários produtos que podem ser obtidos do sensor MODIS, está o produto MOD11 (temperatura superficial e emissividade). Uma das importantes aplicações do produto MOD11 é a validação e melhoria dos modelos de previsão de Tempo. Os dados orbitais usados neste estudo da TS da lagoa Mangueira foram obtidos do produto MOD11 do sensor MODIS. O satélite TERRA foi lançado em dezembro de 1999, coletando dados a partir de fevereiro de 2000. A sua passagem pela América do Sul ocorre as 10:30 da manhã.

As imagens utilizadas no presente estudo foram obtidas durante o mês de dezembro de 2005. Os dados meteorológicos da área de estudo foram obtidos de uma estação de observações superficial em Santa Vitória do Palmar. Esta estação coleta medidas diárias de magnitude e direção do vento em 3 horas distintas do dia; 12h, 18h e 24h. Devido à homogeneidade e topografia da região, as medidas da estação meteorológicas são representativas do campo de vento da Lagoa Mangueira.

\section{DISCUSSÃO E ANÁLISE DOS RESULTADOS}

As Figs. 1 e 2 mostram o perfil de temperatura longitudinal da lagoa Mangueira. Adicionalmente, a Tab. 1 apresenta os dados de velocidade de vento observados na estação meteorológica de Santa Vitória do Palmar. Observando-se os gráficos e a Tab. 1, percebe-se uma relação entre a TS observada da lagoa e a velocidade do vento. A presença de ventos fortes, com direções bem definidas, causa grandes flutuações na TS da lagoa (Fig. 2). Estas 
fortes advecções, com magnitudes variando entre 7 e $9 \mathrm{~m} / \mathrm{s}$ são direcionalmente persistentes. Neste caso, grandes flutuações de temperatura são causadas pela turbulência atmosférica. Este escoamento turbulento de ar, diretamente acima da lagoa, transfere energia para a superficie da água gerando ondas e turbulência. Consequentemente, esta turbulência sobre a superfície da lagoa pode ser o processo fisico responsável por causar as flutuações observadas. Diferentemente, quando o vento é fraco as flutuações de temperatura observada são reduzidas (Fig. 1). Essa análise mostra que o presente método, medindo TS remotas em lagoas representa uma ferramenta efetiva e confiável.

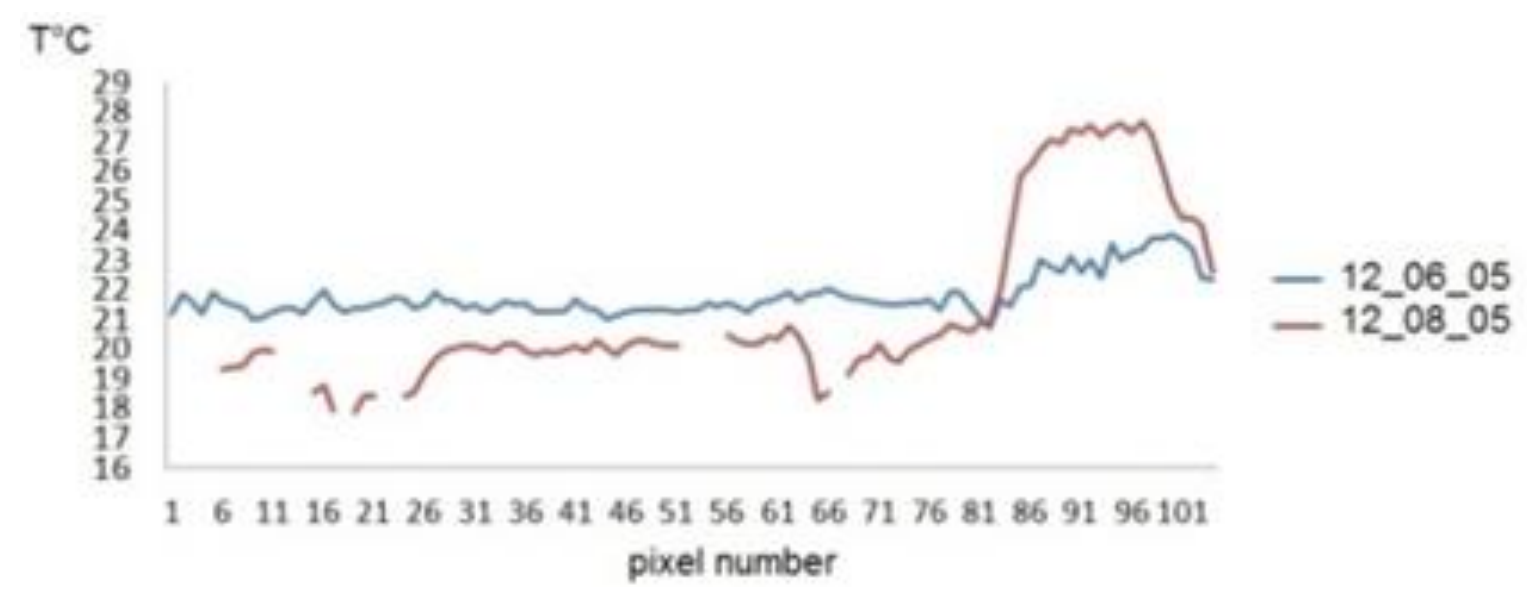

Figura 1. Perfil longitudinal de temperatura para o dia 06 e 08 de dezembro de 2005.

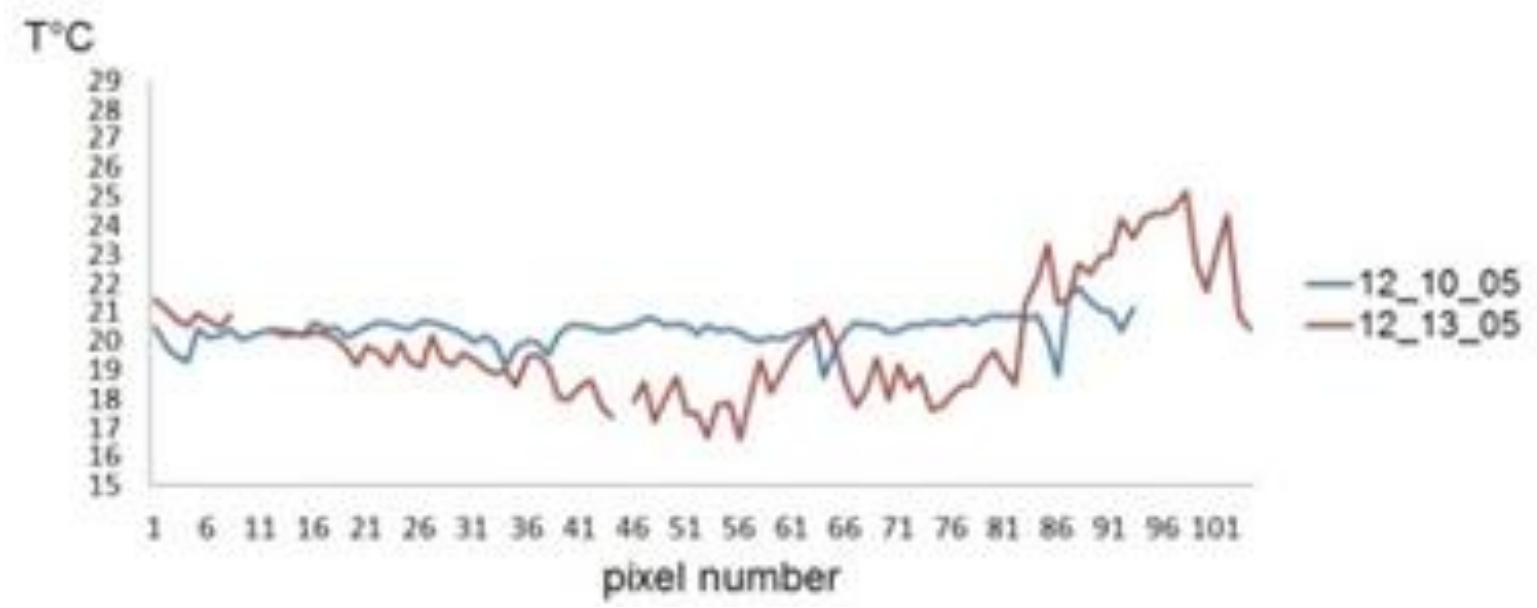

Figura 2. Perfil longitudinal de temperatura para o dia 10 e 13 de dezembro de 2005. 
Tabela 1. Medidas da velocidade do vento.

\begin{tabular}{|c|c|c|c|}
\hline $\begin{array}{c}\text { Imagem } \\
\text { MODIS dos } \\
\text { dias } \\
\text { observados }\end{array}$ & $\begin{array}{c}\text { Hora da } \\
\text { medida de } \\
\text { velocidade }\end{array}$ & $\begin{array}{c}\text { Velocidade do } \\
\text { vento (m/s) }\end{array}$ & $\begin{array}{c}\text { Direção do } \\
\text { vento }\end{array}$ \\
\hline $6 \backslash 12$ & 12 & 5.1 & SW \\
\hline $6 \backslash 12$ & 18 & 6.2 & S \\
\hline $6 \backslash 12$ & 24 & 6.2 & SW \\
\hline $8 \backslash 12$ & 12 & 8.2 & NW \\
\hline $8 \backslash 12$ & 18 & 1.0 & SE \\
\hline $8 \backslash 12$ & 24 & 3.1 & W \\
\hline $10 \backslash 12$ & 12 & 9.8 & S \\
\hline $10 \backslash 12$ & 18 & 8.7 & SE \\
\hline $13 \backslash 12$ & 12 & 8.7 & E \\
\hline $13 \backslash 12$ & 18 & 8.7 & E \\
\hline $13 \backslash 12$ & 24 & 8.7 & E \\
\hline
\end{tabular}

\section{AGRADECIMENTOS}

Os autores agradecem à Capes, CNPq e FAPERGS.

\section{REFERÊNCIAS}

MOHSENI O. AND STEFAN H.G., Water budgets of two watersheds in different Climatic zones under projected climate warming, Climate Change, v. 49, pp. 77-104, 2001.

DELANEY, P.J.V., Physiography and geology of the ar sea of the coastal plain of Rio Grande do Sul, Esp. Esc. Publ. Geol. Univ. Fed. Rio Grande Sul, Porto Alegre, Brazil, v. 6, pp. 1-63, 1965. 